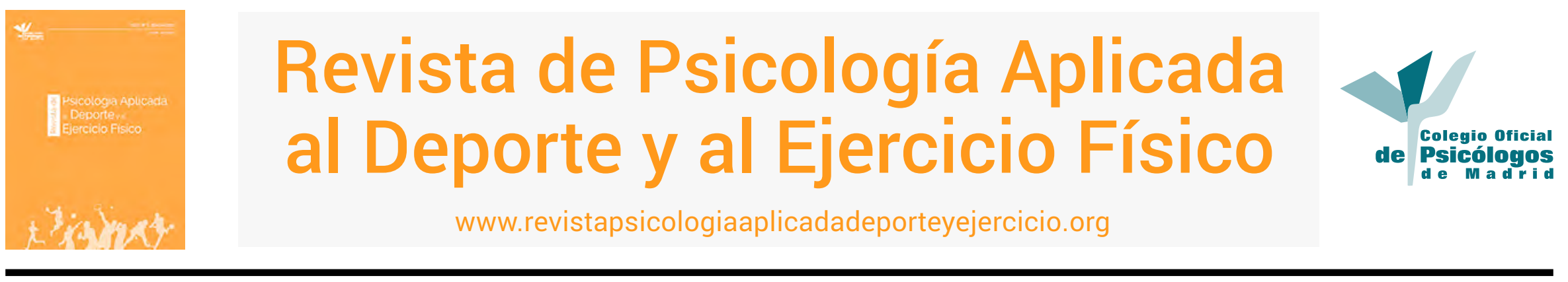

\title{
Intervención psicológica desde el coaching motivacional utilizando el modelo "La Jirafa de Cantón" en una jugadora de
} fúttbol sala

\author{
José Manuel Colás Casaban', Violeta Expósito Boix², David Peris Delcampo ${ }^{1,2}$ y Enrique Cantón Chirivella ${ }^{2}$ \\ 'Associació de Psicologia de l'Esport de la Comunitat Valenciana (APECVA), España, ${ }^{2}$ Universitat de València., España.
}

RESUMEN: En este artículo se presenta la intervención psicológica realizada con una jugadora de fútbol sala de 18 años, perteneciente a la categoría autonómica, que tiene como objetivo principal llegar al fútbol-sala profesional. Para ayudarla en el logro de su meta, se realizan nueve sesiones con una frecuencia de una sesión semanal; en total, dos meses y una semana de duración. Dicha intervención se fundamenta en el modelo de coaching motivacional, concretamente, en la aplicación del modelo "La Jirafa de Cantón", donde el psicólogo genera un entorno facilitador para el autodescubrimiento, autoconocimiento, el establecimiento de objetivos y el desarrollo de un plan de acción. En cuanto a la evaluación de su eficacia, se utiliza un diseño test-retest con los siguientes instrumentos: la Escala de Autoestima de Rosenberg (1965), Escala de Bienestar Psicológico de Ryff (Díaz et. al. 2006) y Escala de Autoeficacia General de Schwarzer y Bassler (1996). Tras la intervención, se constatan mejoras significativas en los resultados de las variables psicológicas medidas: Autoestima, Autoeficacia y las subdimensiones Relaciones Positivas, Dominio del Entorno y Propósito en la Vida pertenecientes al Bienestar Psicológico. Se concluye que el coaching es una estrategia de intervención psicológica eficaz en modificación de la conducta del deportista.

PALABRAS CLAVES: Intervención, deporte, bienestar psicológico, autoestima, autoeficacia.

Psychological intervention from motivational coaching using the "canton's giraffe" model in an indoor football player

\begin{abstract}
This paper reports a psychological intervention carried out with an 18-year-old female who played indoor football in a regional category and whose main objective was to compete in a professional category, that is, two categories higher. To help her achieve her goal, nine sessions were held, one per week, with a total duration of two months and a week. Intervention was based on the motivational coaching model called the "Canton's giraffe", where the psychologist creates an enabling environment for self-discovery, self-knowledge, establishment of objectives, and development of an action plan. Effectiveness of the method was assessed using a test-retest design with the following instruments: Rosenberg Self-esteem (1965); Ryff's Scale of Psychological Well-being (Díaz et al. 2006), and the General Self-efficacy Scale of Schwarzer and Bassler (1996). After the intervention, significant improvements were seen in the results of the psychological variables measured: self-esteem, self-efficacy, perception of control and well-being associated to progress in objectives. It is concluded that coaching is an effective psychological intervention strategy for modifying athlete behavior.
\end{abstract}

KEYWORDS: Intervention, sport, psychological well-being, self-esteem, self-efficacy.

\footnotetext{
José Manuel Colás Casaban es Psicólogo del Torrent CF. Associació de Psicologia de l'Esport de la Comunitat Valenciana (APECVA); Violeta Expósito Boix es Psicóloga. Línea de Motivación, Actividad Física y Salud. Universitat de València; David Peris Delcampo es psicólogo, dirige el Máster Coaching y Liderazgo de la Universitat de València; Enrique Cantón Chirivella es Psicólogo. Director Línea de Motivación, Actividad Física y Salud. Universidad de Valencia. 


\section{Intervenção psicológica a partir do coaching motivacional usando o modelo "la jirafa de cantón” em uma jogadora de futsal}

RESUMO: Este artigo apresenta a intervenção psicológica realizada numa jogadora de futsal de 18 anos, pertencente à categoria regional, cujo principal objetivo é competir dentro de uma categoria profissional, ou seja, promover duas categorias mais elevadas. Para ajudá-la a atingir seu objetivo, são realizadas nove sessões com uma frequência de uma sessão semanal; Sendo no total, dois meses e uma semana. Esta intervenção baseia-se no modelo de coaching motivacional, especificamente, na aplicação do modelo "La Jirafa de Cantón", onde o psicólogo gera um ambiente propício para a autodescoberta, o autoconhecimento, o estabelecimento de objetivos e o desenvolvimento de um plano de ação. Quanto à avaliação de sua eficácia, é usado um teste-reteste com os seguintes instrumentos: Escala de autoestima, de Rosenberg (1965); Escala de bem-estar psicológico, de Ryff (Díaz et al., 2006) e a Escala de Autoeficácia geral, de Schwarzer e Bassler (1996). Após a intervenção, foram observadas melhorias significativas nos resultados das variáveis psicológicas medidas: autoestima, autoeficácia, percepção de controle e bem-estar associado ao progresso nos objetivos. Conclui-se que o coaching é uma estratégia efetiva de intervenção psicológica na modificação do comportamento do atleta.

PALAVRAS-CHAVE: Intervenção, esporte, bem-estar psicológico, autoestima, autoeficácia.

Artículo recibido: 02/09/2017 | Artículo aceptado: 20/11/2017

Martin Seligman, en su discurso de investidura como presidente de la American Psycological Association (APA), definió a la Psicología Positiva como la corriente de la Psicología que pretende desarrollar todo el potencial de las personas y alcanzar el bienestar psicológico, en contraposición de la concepción más clásica que pretende solucionar los problemas de los seres humanos (Seligman, 1999, 2002; Seligman y Csikszentmihalyi, 2000). Desde entonces, se desarrolla esta corriente de la Psicología que pretende adaptarse a las nuevas necesidades de la sociedad actual (Vera, 2008), apoyándose en la investigación científica, buscando entender los procesos psicológicos que subyacen a las emociones, actitudes positivas, cualidades y recursos psicológicos que, por un lado previenen la aparición de patologías y por otro mejoran la salud mental y el rendimiento de las personas (De la Vega, Ruiz-Barquín, Batista, Ortín y Giesenow, 2012).

Enmarcados dentro de la Psicología Positiva, aparecen diferentes términos psicológicos que son objeto de estudio (Ortín, 2017). Entre ellos, cobra especial relevancia el bienestar psicológico eudaimónico, que se refiere a vivir de forma plena en la búsqueda de las propias metas o a buscar la satisfacción derivada de la autorrealización de nuestras potencialidades más valiosas (Ryan, Huta y Deci, 2008), planeamiento relacionado pero diferente a considerarlo como la búsqueda de experiencias positivas minimizando las negativas (Ryan y Deci, 2001). En el bienestar psicológico desde esta perspectiva la persona busca potenciar las cualidades humanas y alcanzar la felicidad. También la autoestima, entendida como un conjunto de pensamientos y sentimientos sobre su propio valor e importancia, es decir, una actitud global positiva o negativa hacia uno mismo (Rosenberg, 1965) o la autoeficacia general, que se refiere a la confianza global que tiene la persona para abordar un am- plio rango de situaciones nuevas o estresantes (Scwarzer y Jerusalem, 1995).

Desde este enfoque orientado hacia la consecución de logros y el desarrollo del bienestar, es posible englobar diferentes estrategias o metodologías de trabajo que se nutren y se pueden enmarcar desde la corriente de la Psicología Positiva (Ortín, 2017). El coaching, entendiendo la diferencia entre una ciencia que es la Psicología y una metodología de trabajo (Cantón, 2014b; Peris-Delcampo, 2014; Caperán y Peris-Delcampo, 2014) se enmarca dentro de esta conceptualización.

Ante la variabilidad de definiciones del coaching existentes, conviene señalar aquellas más aceptadas y que se ajustan más a esta metodología de trabajo.

Para el que quizás es el autor del libro pionero de referencia de este enfoque de trabajo, Timoty Gallwey, el coaching es el arte de crear un ambiente a través de una conversación y con la manera de ser de los protagonistas, que facilita el proceso por el cual una persona se mueve de manera exitosa para alcanzar sus metas soñadas (Gallwey, 2006).

Otra definición ampliamente aceptada señala que el coaching consiste en liberar el potencial de una persona para incrementar al máximo su funcionamiento. Se refiere más a ayudarle a aprender en lugar de enseñarle de la manera más tradicional. (Whitmore, 2003).

Para Villa y Caperán (2010), autores del primer Manual de coaching en España, es aquel proceso de acompañamiento individualizado (coach) en el que el pupilo (coachee) libera su talento a través de la detección de sus puntos fuertes y sus oportunidades de mejora y la elaboración y seguimiento de un plan de acción concreto.

Uno de los autores que ha desarrollado ampliamente el concepto y práctica del coaching en España e Iberoamérica, 
García-Naveira (2013) señala que este consiste en entrenar a una persona o grupo de personas a través de conversaciones eficaces e intencionadas para movilizarla de manera exitosa en la mejora del rendimiento, liderazgo y el logro de las metas establecidas en función de sus necesidades, haciendo hincapié en que el coaching proviene de la Psicología y es una estrategia de modificación de conducta, cuya aplicación representa un proceso de cambio, reflexión, autodescubrimiento, aprendizaje y acción.

Es decir, en conjunto podemos decir que el coaching es un proceso de acompañamiento en el que una persona experta en coaching (llamado coach) crea las condiciones para que el cliente o coachee saque su máximo potencial a través de un proceso de acompañamiento generador de cambio; donde el experto en coaching nunca dice lo que tiene que hacer el coachee, sino que utiliza preguntas, genera situaciones, plantea actividades... para que éste se "dé cuenta" y encuentre sus propias soluciones en busca de objetivos concretos (Peris-Delcampo, 2011a, 2014, 2016, 2017). Además, el experto en coaching (o coach) puede aprender una serie de habilidades de comunicación que le faciliten el generar ese proceso de cambio y ese entorno facilitador, tales como la escucha empática, el generar preguntas de reflexión o acción, el feedback, el silencio, entre otras (Buceta, 2016; García-Naveira, 2011; Peris-Delcampo, 2011b).

Dentro del ámbito del deporte, en el caso del coaching individual, cabe destacar distintas experiencias de intervención con éxito en las que destacan las de García-Naveira (2013) y Peris-Delcampo (2015). Por ejemplo, tras la realización de un proceso de coaching se observa una mejora de la salud y el bienestar psicológico en atletas de rendimiento, tanto en adultos (García-Naveira, 2016) como en adolescentes (García-Naveira, García-Mas, Ruiz-Barquín y Cantón, 2017), como consecuencia del cambio comportamental y la consecución de objetivos, siendo necesario profundizar y establecer modelos psicológicos explicativos.

Desde esta perspectiva metodológica de trabajo, y vista la necesidad de ofrecer modelos y herramientas de intervención desde un coaching bien fundamentado en la Psicología, se creó "La Jirafa de Cantón" (Cantón 2013, 2014a) utilizando una metáfora con un fuerte sustento conceptual y científico, basándose en las teorías de la Motivación de Logro (McCleIland, 1989), el modelo de Autoeficacia (Bandura 1977, 1982, 1997; White 1959), la teoría de la Fuerza Motivacional o modelo de "Expectativas x Valor" (Atkinson 1957, 1983; Tolman 1955; Vroom, 1964) y la Teoría de Metas (Deci y Ryan 1985; Dweck, 1991; Ford 1992; Locke 1968, Locke y Latham 1990; Tolman, 1955). Todos ellos se desarrollan ampliamente en los diferentes manuales de Motivación y Emoción (Palmero, Fernández-Abascal, Martínez y Chóliz 2001; Reeve 2010).
Dicho modelo (La "Jirafa de Cantón") se ha mostrado eficaz en diferentes entornos (Peris-Delcampo, Expósito y Cantón, 2016), habiéndose desarrollado un protocolo de intervención (Cantón y Peris-Delcampo, 2017) que facilita su aplicación, tanto para los profesionales de la Psicología como para otros que, desde su específico rol profesional, emplean las estrategias de coaching para la gestión de las personas en su puesto de trabajo (Cantón, 2014b). Asimismo, se pudo comprobar que manejando adecuadamente aspectos motivacionales, es posible mejorar el rendimiento de deportistas, como por ejemplo la toma de decisiones de jugadores de voleibol en función de sus años de experiencia en la práctica deportiva (León, Boix, Serrano y Paredes, 2017).

El presente trabajo es un estudio de caso único, con un diseño pre y post intervención en coaching, utilizando el modelo de "La Jirafa de Cantón" a una jugadora de fútbol sala. El principal objetivo es determinar la eficacia de la intervención, en el que se espera una mejora de las variables psicológicas evaluadas (autoestima, bienestar psicológico y autoeficacia general).

\section{Método}

\section{Participantes}

La participante de 18 años es una jugadora de la categoría autonómica federada de fútbol sala. Lleva practicando este deporte desde hace 10 años en equipos diferentes. Entrena dos días a las semana, de 19:30 a 21 horas y el fin de semana tiene un partido de competición o amistoso.

La intervención fue realizada por un psicólogo especializado en deporte (Máster en Psicología del Deporte) experto en el empleo del modelo "La Jirafa de Cantón".

Antes de realizar la intervención, se le informó a la participante de las condiciones de la intervención dando su consentimiento para la utilización de los datos para fines de investigación manteniendo la confidencialidad necesaria.

\section{Objetivos Generales y Específicos}

El objetivo general de la intervención es ayudar a la deportista (coachee) a lograr su meta (participar en un equipo que compita a nivel nacional) interviniendo sobre los procesos motivacionales a través de una metodología no directiva (coaching) que, entre otras cosas utiliza preguntas en lugar de respuestas (Peris-Delcampo, 2011a, 2017). Para la consecución de la meta, se establecieron una serie de objetivos específicos que se detallan a continuación: 
- Obtener información sobre los componentes de la motivación implicados.

- Detectar las fortalezas y carencias en los procesos motivacionales.

- Favorecer la reflexión sobre posibles estrategias de consecución de la meta a través de preguntas.

- Fomentar la participación activa y el compromiso de la persona en su proceso de cambio y mejora.

- Evaluar la eficacia de la intervención a través de las variables de autoestima, bienestar psicológico y autoeficacia, en las que se espera un incremento con respecto a la línea base (medida realizada antes de la intervención).

- Evaluar la eficacia de manera cualitativa mediante la percepción subjetiva sobre la intervención realizada.

\section{Instrumentos}

En primer lugar, se utilizó la Escala de Autoestima de Rosenberg (Rosenberg, 1989), cuyas características psicométricas han sido desarrolladas ampliamente en su versión original americana y en otros idiomas entre los que está el español, con un alpha de Cronbach de .80 (Vázquez Morejón, Vázquez-Morejón Jiménez y Bellindo Zanin, 2013; Vázquez Morejón, Jiménez García-Bóveda y Vázquez-Morejón Jiménez, 2004). La escala está compuesta por 10 ítems con un formato de respuesta tipo Likert, con puntuaciones desde 4 ("Muy de acuerdo") a 1 ("Muy en desacuerdo"). La autoestima es entendida como un sentimiento de valor personal y respeto hacia uno mismo.

Además, se ha utilizado la Escala de Bienestar Psicológico de Ryff, en su adaptación española (Díaz et al., 2006), la cual mostró un alpha de Cronbach entre .84 a .70 según sus dimensiones. La escala está formada por 29 ítems con un formato de respuesta tipo Likert, con puntuaciones desde 1 (Completamente en desacuerdo) a 6 (Completamente de acuerdo). Las dimensiones de la escala son:

- Auto-aceptación: hace referencia a que la persona tenga una actitud positiva hacia sí misma y sea consciente de sus propias limitaciones facilita un funcionamiento psicológico positivo.

- Dominio del entorno: definido como la habilidad de crear o elegir entornos favorables para el cumplimiento de las necesidades y de los objetivos personales, lo cual se asocia a una mayor percepción de control e influencia sobre el mundo que les rodea sintiéndose capaces de influir en su contexto vital.

- Relaciones positivas: se refiere a que la persona mantiene relaciones sociales estables, maduras y de con- fianza con los otros. Esta dimensión es un componente fundamental para la salud mental (Ryff, 1989ab).

- Propósito en la vida: mide el grado de claridad de las metas y objetivos vitales. El funcionamiento positivo óptimo requiere el empeño por desarrollar sus potencialidades.

- Crecimiento personal: entendido como la capacidad de la persona para crear situaciones y condiciones que le permitan desarrollar su potencial, por seguir creciendo como persona y llevar al máximo sus capacidades (Keyes, 2002).

- Autonomía: evalúa la capacidad de mantener la propia identidad en los diferentes contextos sociales donde se ve inmersa la persona. Esto es característico en personas que son capaces de resistir en mayor medida la presión social y auto-regulan mejor su comportamiento (Ryff y Singer, 2002).

Así mismo, se ha empleado la Escala de Autoeficacia General (Schwarzer y Bassler, 1996), en su adaptación española de Sanjuán, Pérez-García y Bermúdez (2000), que además cuenta con otra adaptación española (Vázquez Morejón et al., 2004), presentando un alpha de Cronbach de .83. La escala está compuesta por 10 ítems, los cuales se valoran en función de una escala de respuesta tipo Likert de cuatro puntos, siendo 1 ("Poco de acuerdo") y 4 ("Muy de acuerdo"). La autoeficacia general es entendida como el sentimiento estable de competencia personal para manejar de forma eficaz una gran variedad de situaciones estresantes.

\section{Procedimiento y Diseño de la Intervención}

El modelo de "La Jirafa de Cantón" (Cantón 2013, 2014a) está enfocado desde la perspectiva del coaching y pretende generar un proceso facilitador entre el psicólogo experto en coaching y la persona que se beneficia del proceso o coachee. Se estructura en procesos motivacionales con niveles sucesivos, lo cual permite identificar las fortalezas o debilidades de los componentes motivacionales para potenciar la acción y la reflexión de la persona sobre los mismos. El modelo identifica cuatro niveles o elementos fundamentales (ver Figura 1) que se identifican y analizan a través de una serie de preguntas (ver Anexo 1).

1 La cabeza: Esta parte ilustra metafóricamente el logro u objetivo a conseguir y hacia el que se orienta, y que debe estructurarse en pasos sucesivos, además de plantearse de forma flexible, específica, concreta y operacionalizada. A la vez, debe orientarse a la tarea, es decir, ha de depender de la persona, y suponer un reto alcanzable. 


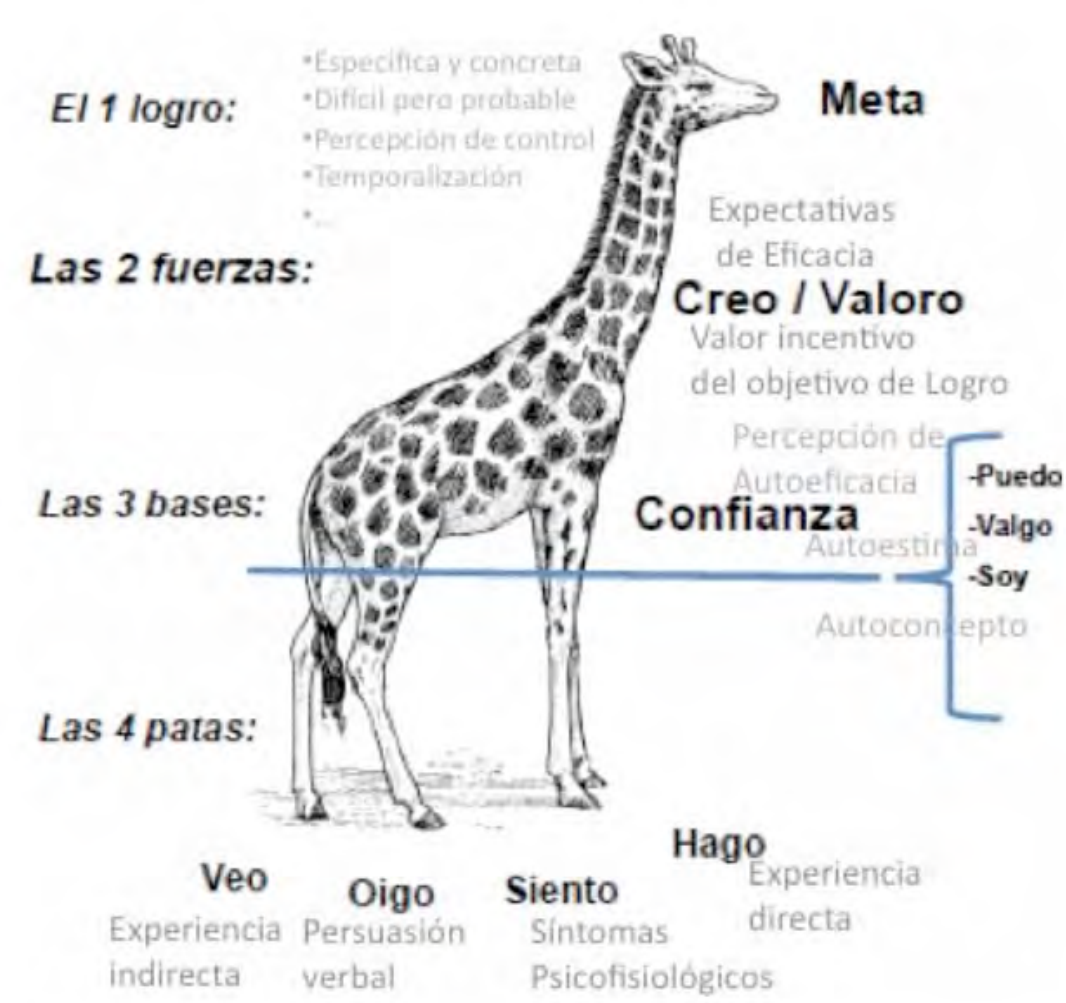

Figura 1. Modelo motivacional desde el enfoque coaching "La Jirafa de Cantón" (Cantón, 2013, 2014a).

Así mismo, la persona debe estar comprometida con su objetivo y contar con un apoyo social propicio para su consecución.

2 Cuello: Los procesos motivacionales generan una base que nos permite avanzar hacia una meta, reconocido como fuerza motivacional (Atkinson, 1957, 1983; Bandura, 1997; Vroom, 1964), que es la combinación de dos variables: las expectativas de eficacia (lo que creo que soy capaz de hacer) y el valor incentivo (intrínseco o extrínseco) del objetivo de logro (lo que valoro aquello que quiero conseguir). Ambas variables (expectativa y valor) generan una fuerza impulsora de la motivación que aumenta la probabilidad de lograr la meta.

3 Cuerpo: Esta parte hace referencia a la autoconfianza que se desarrolla basándose en la percepción de la autoeficacia (puedo), el concepto de autovalía (valgo) y de su autoconcepto (soy). A la vez, facilita focalizarse en los puntos fuertes de uno mismo, dado que le han trascendido en una valoración positiva sobre sí mismo. En la figura 1 se representa la percepción de autoeficacia como "puedo", la autoestima como "valgo" y el autoconcepto como "soy".

4 Patas: Representan las cuatro vías de información en las que se apoya la autoconfianza y el desarrollo de la motivación intrínseca (Reeve 2010), las cuales son: la experiencia directa (mi propia experiencia de vida), la experiencia indirecta, vicaria u observacional (lo que de mí veo reflejado en los demás), la persuasión verbal (mensajes de los demás y el autodiálogo) y la interpretación de los síntomas psicofisiológicos tanto en un sentido corporal como emocional.

Con el objetivo de evaluar la eficacia del modelo, se utiliza un diseño A-B-A (Frías, Pascual y García, 1999). Este método se centra en una primera fase de recogida de los datos del deportista mediante una medición cuantitativa del nivel inicial de autoestima, autoeficacia y bienestar psicológico $(A)$, posteriormente se trabaja utilizando la estrategia de coaching motivacional "La Jirafa de Cantón" (B) y, finalmente se realiza una segunda recogida de datos del deportista (A) en la que se espera obtener una mejora en las variables analizadas mediante tres cuestionarios, que se detallan en el siguiente apartado.

Según un protocolo diseñado para este modelo (Cantón y Peris-Delcampo, 2017), en la fase B de trabajo con el modelo "La Jirafa de Cantón" se divide en dos subfases: una de identificación en la que se determina dónde está el sujeto o coachee respecto a cada una de las partes del modelo; y otra de trabajo específico o de intervención donde se trabaja las carencias o potencialidades de cada una de las partes de la "Jirafa de Cantón" para fortalecerlas en función del objetivo o meta propuesta.

En el Anexo 1 se detallan algunos ejemplos de las preguntas (desde el enfoque coaching) utilizadas para la identificación y desarrollo de cada una de las fases del modelo.

La intervención realizada con la jugadora de futbol sala se ha desarrollado en nueve sesiones, iniciando el 26 de abril de 2017 y finalizando el 14 de junio de 2017. Las nueve sesiones totales de la intervención, tuvieron una duración media de una hora.

En primer lugar, se presentan las sesiones de forma visual (Tabla 1) para después desarrollarlas más detalladamente.

$1^{\text {a }}$ sesión. 26/04/2017. En esta sesión se centró en la explicación de los aspectos psicoeducativos del modelo y se realizó el pase inicial de los cuestionarios (fase pre test).

$2^{a}$ sesión. 03/05/2017. Se comienza el trabajo de orientación al cambio mediante una entrevista semiestructurada con el objetivo de tener un ritmo fluido durante la misma y, a la vez, ir desglosando las partes del modelo "la Jirafa de Cantón". Se inicia el modelo con la cabeza (objetivo a conseguir) que cumple los requisitos para generar una motivación adecuada. Se destaca que, desde un principio, la meta era específica y estaba operacionalizada, pues según afirma la jugadora, sus compañeras y antiguos entrenadores reconocen que destaca física y técnicamente, pero requiere mejorar mentalmente, puesto que esto no le permite, en muchas ocasiones rendir al máximo nivel, como por ejemplo, colocarse bien tácticamente. 
Tabla 1. Estructura de la intervención con la jugadora de fútbol sala desde el modelo "La Jirafa de Cantón"

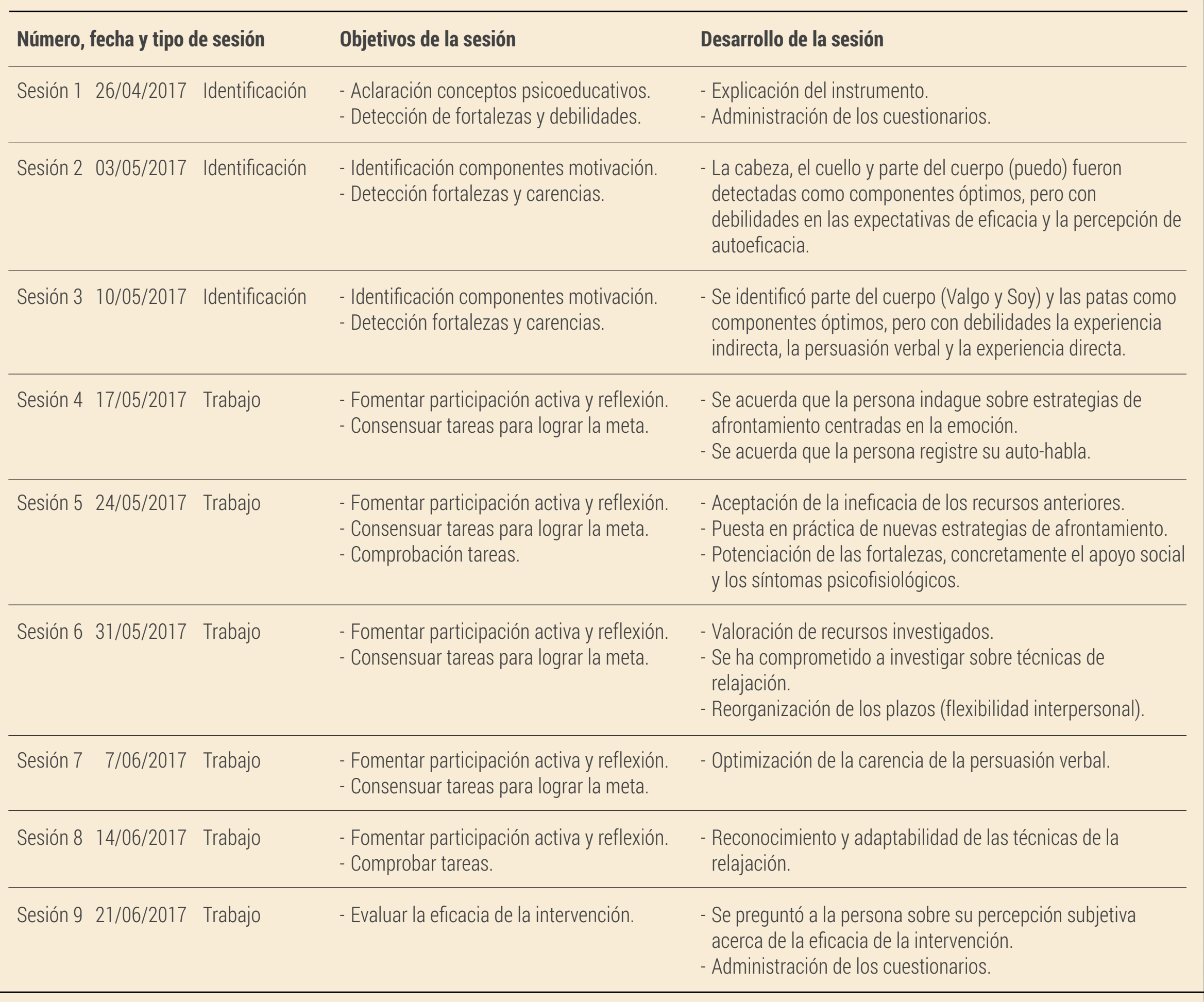

La jugadora es una persona estructurada, se organiza bien. También, es flexible, permitiéndose ajustes en función del criterio de logro, por ejemplo, "si en lugar de salirme del partido pido el cambio para volver entrar estaría muy bien". Así mismo, se registran puntos de evaluación aprovechando el calendario y el torneo post-temporada "en la ida ellas eran muy sucias y tras insultarme dos veces me revolví y la empuje". En cuanto al apoyo social, lo recibe fundamentalmente de su madre y su hermano, ya que "siempre que vienen me vengo arriba y tengo más fuerza, y cuando me dan una patada o me empujan o algo, me animan y es más fácil seguir el juego".

Se continúa identificando el estado de las fuerzas motivadoras y, en la parte del cuello, la jugadora explica que no tiene expectativas de eficacia, en otras palabras, afirma que "ante partidos muy competidos con pocos fallos me salgo" a causa de que "me cuesta mucho tener fallos en partidos importantes". En cambio, indica que valora su meta de diez sobre diez, ya que es una cuestión que "en varias ocasiones me ha llevado a no estar en equipos más competitivos". Por ello indica que "si consiguiera seguir jugando sería la leche", puesto que es el deporte que más le apasiona, además de que "superarlo y que lo viera mi madre sería muy bonito", por tanto, conseguirlo le aportaría una gran satisfacción personal.

Se continúa con la confianza (cuerpo) y se detecta que, en la percepción de autoeficacia, "puedo", la jugadora quiere conseguir su meta más allá de las adversidades que puedan presentarse incluso si no es en el plazo establecido no 
pasaría nada ("necesito mantenerme viva en el partido"). Además, se muestra con confianza en la consecución del objetivo, en gran parte por su autonomía y el apoyo de sus más allegados.

$3^{\text {a }}$ sesión. 10/05/2017. Esta sesión se inicia con el análisis de la parte "valgo" (autoestima). Se observa que tiene una autoestima muy alta, que se ve capaz de conseguir el reto que se presenta muy desafiante, aunque reconoce que hasta ahora no ha podido y "le va a costar mucho esfuerzo".

En relación al autoconcepto ("soy"), reconoce que una cualidad que la define es "el nervio que tengo" que le facilita durante el juego a "realizar jugadas que hasta yo misma me digo: ¡qué buena eres!" pero a veces me juegan malas pasadas. Otras cualidades que facilitan es la pasión por practicar fútbol sala a pesar de las adversidades, por ejemplo "vengo de estudiar o de trabajar y yo a entrenar, nunca fallo" o "haga el tiempo que haga yo vengo". Por ello, el autoconcepto de esta jugadora se reconoce por la "perseverancia, trabajo para conseguir lo que quiero a pesar de las dificultades, soy autoexigente, extrovertida y visceral".

Posteriormente, se identifican las cuatro "patas" o vías de generación de confianza. En primer lugar, la persuasión verbal ("lo que oigo") no está lo suficientemente fuerte porque en su círculo más cercano en el campo (su equipo) cada vez que la jugadora se muestra "súper activa" mandan mensajes negativos "no la líes" o "iqué empujón te ha dado la 9!". En cambio, en la grada con la presencia de sus familiares o amigos, recibe mensajes de ánimo, facilitando pensamientos de éxito frente al objetivo, potenciando la percepción de eficacia. En referencia al autodiálogo, es positivo si están en la grada sus familiares ("tengo que seguir") pero, en el caso contrario, los mensajes son negativos ("me pongo muy nerviosa"). En las próximas sesiones se retoma la funcionalidad de esta pata, considerada débil.

En cuanto a la experiencia indirecta ("lo que veo"), tiene amigas que compiten en ligas superiores, conoce a entrenadores que están en ligas superiores y considera que tiene figuras de referencia cercanas. Sin embargo, reconoce que nunca les ha preguntado que recursos tienen o estrategias utilizan para conseguir el objetivo ni su experiencia personal. En las próximas sesiones, se retomará la funcionalidad de esta parte.

En referencia a los síntomas psicofisiológicos ("lo que siento") se expresa con seguridad, mostrando una pata óptima, dado que la jugadora razona el objetivo desde la superación, el orgullo y la alegría. Así mismo, reconoce que para su familia sería una alegría también y eso le emociona más.

Por otro lado, la experiencia directa ("lo que hago") se considera que es la parte más débil, puesto que es la que se ve y sus compañeros de equipo reconocen. Reconoce que su en- trenador, muchas veces cuando le pide el cambio para irse no le hace caso y eso le altera más. Las próximas sesiones se centrarán a fondo con esta pata, ya que está muy necesitada de desarrollo y refuerzo.

$4^{\text {a }}$ sesión. 17/05/2017. En esta sesión la jugadora se centró en las tareas propuestas en la sesión anterior, focalizadas en buscar información sobre las estrategias que usan sus conocidos que compiten en ligas superiores para no salirse del partido con reacciones emocionales, autorregulación emocional.

En este aspecto, aportó información sobre experiencias indirectas ( $1^{\circ}$ tarea). La jugadora reconoce "no me imaginaba que ellas se alteraran tanto porque no se les nota", y le afirmaron "si no mantengo la cabeza en el juego pierdo el sitio", Por estas y más justificaciones, exterioriza que ahora cuenta con más recursos para afrontar situaciones posibles, así pues, indica que puede empezar a probarlas en los entrenamientos de esta semana e incluso en el partido del fin de semana, ya que el encuentro en la ida fue muy tranquilo ( $2^{a}$ tarea).

En referencia, al autodiálogo reconocido como negativo sin presencia de sus familiares y que impedía obtener una percepción de eficacia optima de los propios recursos frente a las adversidades. Se ha comprometido a recoger información sobre los mensajes emitidos en los próximos entrenamientos o partido del fin de semana ( $3^{a}$ tarea).

Además, la jugadora, animada por uno de sus antiguos entrenadores, se ha comprometido a buscar información sobre noticias de jugadoras o jugadores que demuestran autorregulación emocional en público ( $4^{\mathrm{a}}$ tarea).

Durante esta semana, en relación a las sesiones de entrenamientos, la jugadora indica que se ha suspendido una sesión debido a un número elevado de ausencias de las compañeras de equipo. Se muestra frustrada, ya que explica que los motivos de las compañeras no son razonables y que ella los relaciona más con los resultados del fin de semana. Sin embargo, ella sigue con la misma confianza, por lo que estos contratiempos no afectan a su motivación para conseguir la meta ni a los plazos que se ha propuesto. Al mismo tiempo, considera que esto le puede beneficiar con la respuesta que tuvo, preparándose físicamente con mayor intensidad.

$5^{a}$ sesión. 24/05/2017. En esta sesión, se comprobó la $2^{a}$ tarea relacionada con las estrategias de afrontamiento de sus amigas, quienes compiten en otras categorías superiores, ante posibles reacciones emocionales incontroladas. Ella reconoce que hay una estrategia en concreto que le ha sido útil esta semana, durante las sesiones de entrenamiento, como recursos frente momentos de enfado o de frustración. Se agachaba y se ataba más fuerte los cordones a la 
vez que utilizaba el autodiálogo ("activa") con la intención de seguir rindiendo en los ejercicios programados.

En referencia a la $3^{a}$ tarea, la jugadora presentó la información recogida sobre el diario de autodiálogo con mensajes considerados negativos ("no puedo", "me voy", "me cambio" y "me salgo") que causaban comportamientos disfuncionales o inadaptativos, los cuales impedían optimizar la percepción de eficacia.

Así mismo, la jugadora al explicar el registro, mostró con una actitud reflexiva mientras indicaba las circunstancias de dichos momentos y también que aprovechaba estos instantes para salir o pedir el cambio, a sabiendas que eso le conllevaba a salir de la práctica deportiva. En otras palabras, reconoce utilizar el autodiálogo negativo como estrategia de afrontamiento cognitiva y de evitación sobre la emoción con el fin de distanciarse de la intensidad de la emoción producida.

También señala que ella no se divierte cuando suelen pasar este tipo de cosas, en cambio, disfruta verdaderamente cuando sus familiares le animan a pesar de los errores o las posibles adversidades. Por ello, se le indaga si recuerda los mensajes les suelen decir, y reconoce que su fuente de apoyo le proporciona mucha ayuda. Con ello, se le pregunta si podría relacionar los mensajes que recuerda, propios de la fuente de apoyo, con sus respuestas antes citadas. Ella afirma y expone que, cuando está más tranquila, es más factible y que lo intentará llevar a cabo esta semana. Se le sugiere si podría registrarlo, con el fin de conocer la adaptabilidad de las consecuencias (5 $5^{a}$ tarea).

$6^{\text {a }}$ sesión $31 / 05 / 2017$. Esta sesión comienza con la comprobación de la información recogida sobre estrategias de autorregulación de jugadoras profesionales (4a tarea) Esta iniciativa fue recomendada por un antiguo entrenador. Ella dice que ha visto muchos vídeos montados con música pero que realmente no lo ve útil, dado que, son muy cortos y se ven reacciones de deportistas. Además, según comenta, las conductas se ven de forma inverosímil pues son reacciones muy diferentes a como le gustaría a ella. Aun así, expone que, si muchos deportistas utilizan estrategias de relajación con el fin de controlar esos momentos concretos, ella puede buscar estrategias que se adapten más a sus necesidades (6 tarea).

La jugadora explicó sus sensaciones del encuentro del fin de semana, ultimo de la liga, que fue tranquilo y que cada vez se divierte más compitiendo con el equipo. Disfruta de más minutos, pero indica que quiere trabajar durante más tiempo, alargando las fechas previstas para conseguir su objetivo a largo plazo focalizándose en un trabajo predeterminado que potencie el control emocional, ya que se ha dado cuenta de que en función de cómo reaccionaba ante el enfado o la frustración cuando jugaba, le producía una bajada de su rendimiento. Además, concreta con "defendía tarde y atacaba mal", "pedía el cambio", "jugaba como si el partido no iba conmigo" y ahora "estoy llegando bien muchas veces", "estoy disfrutando con más calidad el tiempo que juego", "acabo los partidos". Por eso "cuando juego, estoy más pendiente de cómo llegar y tengo buenas sensaciones, como si estoy mejorando por pasos".

Por otro lado, indicó que después de disputar el último partido del campeonato de liga iba a aprovechar que, en dos semanas daría comienzo el torneo de la "copa futsal". Siente pena que finalice la temporada en estos momentos pero que ahora reconoce que requiere más tiempo para llegar a conseguir recursos más sólidos para lograr su objetivo. Admite que está adquiriendo recursos que le están siendo útiles para ir mejorando poco a poco. En este sentido expone que, "antes pensaba que yo controlaba las situaciones de enfado por que me centraba en que se me pasara y no en el rendimiento que ofrecía".

$7^{a}$ sesión $07 / 06 / 2017$. Esta sesión se inicia con la $5^{a}$ tarea centrada en la relación del registro de las expresiones, que se suele decir ella misma tras situaciones de enfado o frustración, con las siguientes situaciones con el fin de conocer la adaptabilidad de su comportamiento. Tras comentarlo, ella considera que su comportamiento no le ayuda a mantenerse centrada en el juego y que cuando le animan, es más fácil continuar. En otras palabras, según se explicaba podemos interpretar que con sus mensajes de autodiálogo facilitaba un anclaje emocional y con los mensajes de apoyo y ánimo se facilitaba una actitud más fluida emocionalmente.

En consecuencia, reconoce que el autodiálogo basado en mensajes centrados en el ánimo y el apoyo aumenta su rendimiento, disminuyendo las reacciones emocionales incontroladas. Este recurso, según explica lo considera muy valioso y movilizador, pues le permite seguir funcionando.

Después, se continúa la sesión con la $6^{a}$ tarea centrada en el desarrollo de la cognición, de que, si muchos deportistas utilizan estrategias de relajación, con el fin de controlar los momentos críticos, puede registrar estrategias que se adapten más a sus necesidades. Con el registro realizado, indica que le parecen interesantes el control de la respiración, la relajación progresiva y la música. El siguiente paso se centra en delimitar sus momentos críticos englobando "cuando cometo un fallo, que decida una jugada o un partido" o "cuando recibo en alguna acción algún tipo de violencia verbal o física". Para la siguiente semana (primer fin de semana del torneo) se acuerda prepararlas, practicarlas y registrar el resultado para comprobarlo en la siguiente sesión. 
$8^{a}$ sesión 14/06/2017. En esta sesión, se continúa con el desarrollo de la $6^{a}$ tarea con el registro de la puesta en práctica de las diferentes estrategias de relajación. Ella expone que la relajación progresiva en una semana la ha practicado una vez antes del fin de semana y que no ha encontrado mejoría. Preguntando la eficacia que tiene en otros deportistas llegamos a la conclusión que es una técnica que requiere mayor tiempo de entrenamiento o práctica.

En cuanto a la respiración indica que es una técnica muy útil que la ha utilizado en varias ocasiones pero que, en los partidos, una vez llegado el momento de jugar, no lo ve factible. Quizá "al principio de los encuentros sí, o al inicio de una acción técnica también, bien sea en una acción a balón parado, o bien ante un regate o una temporización", pero explica que tras un fallo le cuesta controlar respiración para autorregularse emocionalmente.

En cambio, con la música, tras una situación crítica considera que se centra en "alguna canción y me posiciono con mayor control de la situación, entiendo que ante un momento de mucho nervio a la vez puedo atarme los cordones con intensidad, o emitir mensajes de autohabla funcionales". Además, afirma que al jugar el torneo "había música ambiental y me resultaba fácil guiarme con la música porque estaba ahí, no tenía que imaginármela". Pero "en otras ocasiones sí que lo utilizaré, aunque no esté".

Con el desarrollo del modelo y volviendo al estado adaptativo de "las patas", podemos interpretar que las vías de influencia han modificado el impacto sobre la conciencia de los recursos de afrontamiento sobre la emociones. Ahora explica que está más cerca de autorregularse emocionalmente frente a situaciones críticas, entiende lo que los demás ven en ella cuando reaccionaba de esa forma. Sigue considerando sus puntos fuertes, pero en sus debilidades entiende que ahora está trabajando de forma más eficaz que antes. Aunque le lleve tiempo será capaz de conseguir su objetivo, se siente con más confianza ahora que respecto al inicio aumentando la percepción de logro de su objetivo. ga sesión. 21/06/2017

Tras la aplicación del modelo de "La Jirafa de Cantón", se procedió a realizar otra evaluación con los cuestionarios anteriores (fase de post test), para ver si se ha producido algún impacto en el sujeto que se traduzca en cambios en las puntuaciones en los mismos.

Además, en la sesión se aprovechó para comentar la evolución del proceso y valorar la percepción de la jugadora en relación a los avances obtenidos. Se mostró satisfecha por la intervención realizada y más segura a la hora de pensar en su rendimiento y posibilidades para la temporada siguiente.

\section{Resultados}

A continuación, se presentan los resultados de la evaluación pre y post intervención (Tabla 2).

\begin{tabular}{|c|c|c|c|}
\hline Cuestionario & Pre test & Post test & Diferencia \\
\hline Autoestima & 32 & 38 & +6 \\
\hline \multicolumn{4}{|l|}{ Bienestar Psicológico } \\
\hline Autoaceptación & 23 & 21 & -2 \\
\hline Relaciones Positivas & 27 & 29 & +2 \\
\hline Autonomía & 35 & 34 & -1 \\
\hline Dominio del Entorno & 24 & 28 & +4 \\
\hline Crecimiento Personal & 24 & 24 & 0 \\
\hline Propósito en la vida & 27 & 28 & +1 \\
\hline Autoeficacia general & 32 & 36 & +4 \\
\hline
\end{tabular}

En la fase de pre - intervención, respecto a la Autoestima, obtiene una puntuación de 32 sobre 40 . En cuanto al Bienestar Psicológico, alcanza unos valores de 23 sobre 24 para la dimensión de autoaceptación, unos valores de 27 sobre 30 para la dimensión de relaciones positivas, unos valores de 35 sobre 36 para la dimensión de autonomía, unos valores de 24 sobre 30 para la dimensión de dominio del entorno, unos valores 24 sobre 24 para la dimensión de crecimiento personal y unos valores de 27 sobre 30 para la dimensión del propósito en la vida. Y por último, la jugadora presenta una Autoeficacia General de 32 sobre 40.

En la fase de post - intervención, respecto a la Autoestima, obtiene una puntuación de 38/40. En cuanto al Bienestar Psicológico, alcanza unos valores de 21/24 para la dimensión de autoaceptación, unos valores de 29/30 para la dimensión de relaciones positivas, unos valores de 34/36 para la dimensión de autonomía, unos valores de 28/30 para la dimensión de dominio del entorno, unos valores $24 / 24$ para la dimensión de crecimiento personal y unos valores de 28/30 para la dimensión del propósito en la vida. Y por último, la jugadora presenta una Autoeficacia General de 36 sobre 40

Respecto a las diferencias en función de las puntuaciones en general, en el cuestionario de Autoestima de Rosenberg se obtuvo una puntuación superior por un valor de 6 . En el cuestionario de Bienestar Psicológico de Ryff en la dimensión de autoaceptación se alcanza un valor inferior por 
2 puntos, como también en la dimensión de autonomía se sitúa en 1 punto por debajo. En cambio, en la dimensión de relaciones positivas logra aumentar en 2 puntos, en la dimensión del dominio del entorno aumenta en 4 puntos y en la dimensión del propósito en la vida aumenta en 1 punto. Y por último en cuestionario de la Autoeficacia General obtuvo una puntuación superior en 4 puntos.

En el cuestionario de Autoestima de Rosenberg, se puede observar que los ítems que experimentaron los cambios más significativos son:

- Ítem 8. Me gustaría poder sentir más respeto por mí mismo. (3 puntos). Este ítem sube de 1 punto a 3 puntos, en otras palabras, la deportista se percibe con mayor respeto aumentando su frecuencia experiencial y ha disminuido significativamente las situaciones con necesidad de percibirse con mayor respeto.

- Ítem 10. A veces creo que no soy bueno/a persona. (3 puntos). Este ítem sube de 1 punto a 3 puntos, por ende, la deportista se percibe emocionalmente más adaptada ante las diferentes situaciones y ha disminuido significativamente la percepción de emociones no saludables.

En el cuestionario de Bienestar Psicológico de Ryff, las diferencias más significativas en los ítems residen en la variaciones de las siguientes dimensiones:

- Autoaceptación: Las variaciones se han registrado en los ítems 1 ("Cuando repaso la historia de mi vida estoy contento con cómo han resultado las cosas"), 17 ("Me gusta la mayor parte de los aspectos de mi personalidad") y 24 ("En su mayor parte, me siento orgulloso de quien soy y de la vida que llevo"); disminuyendo en 1 punto cada uno, aun así, se mantienen las puntuaciones en un rango alto de autoaceptación. Tras la intervención, ha aumentado la autoconsciencia tanto de los recursos óptimos como de los que se deben mejorar.

- Relaciones positivas: Las variaciones en esta escala se han registrado localizadas en el ítem 12 ("Siento que mis amistades me aportan muchas cosas") aumentado en 2 puntos. El cambio originado en la percepción de las relaciones sociales es interpretado en función del enriquecimiento de las mismas al optimizar el grado de bienestar como consecuencia de la intervención con el modelo "La Jirafa de Cantón".

- Autonomía: Las variaciones en esta escala se han localizado en el ítem 23 ("Es difícil para mí expresar mis propias opiniones en asuntos polémicos"), disminuyendo en 1 punto. El cambio producido en la percepción de la capacidad para autorregular su conducta frente al grupo, es interpretado en base al reconocimiento de los objetivos personales y de las consecuencias reales. Por ende, se asume la posibilidad de optimizar la co- municación con los demás con el fin de acceder a las metas personales. Esta modificación de perspectiva de los recursos utilizados para la consecución de logros personales se ha facilitado con la intervención.

- Dominio del entorno: Las variaciones en esta escala se han delimitado significativamente en los ítems 5 ("Me resulta difícil dirigir mi vida hacia un camino que me satisfaga") y 29 ("Si me sintiera infeliz con mi situación de vida daría los pasos más eficaces para cambiarla"), aumentando el primero en 4 puntos y el posterior en 2 puntos. El cambio más significativo se ha producido en la percepción de control sobre el entorno. Esta modificación es interpretada en función de la declaración de situaciones conflictivas que impedían mantener una actitud activa para el logro de los objetivos personales y la búsqueda de recursos propios más adecuados para mantener dicha actitud. Por consiguiente, se asume la posibilidad de optimizar la propia actuación ante las situaciones conflictivas con el fin de mostrarse más activo en la consecución de las metas personales. Esta búsqueda de recursos óptimos frente a situaciones conflictivas ha mejorado gracias a la aplicación de "La Jirafa de Cantón"

- Propósito en la vida: Las variaciones en esta escala se han originado en los ítems 15 ("Me siento bien cuando pienso en lo que he hecho en el pasado y lo que espero hacer en el futuro") y 20 ("Tengo clara la dirección y el objetivo de mi vida"), disminuyendo el primero en 1 punto y el posterior aumentando en 2 puntos. Asimismo, la primera modificación se ha producido en la introspección de actuaciones pasadas frente a situaciones anteriores, donde las sensaciones no han sido agradables al entender que han sido oportunidades perdidas en el logro de las metas personales. En cambio, la segunda modificación se ha producido con la búsqueda de recursos personales que optimicen el rendimiento mostrado. Dado que, ahora el objetivo obtiene mayor clarividencia con el reconocimiento de los propios recursos que posibilitan incrementar el anterior rendimiento. Esta modificación genera en la deportista mayor luminiscencia en sus recursos para la consecución de su objetivo.

Y por último, en el cuestionario de Autoeficacia de Schwarzer y Baessler destacamos los ítems que experimentaron los cambios más significativos:

- Ítem 2. Puedo resolver problemas difíciles si me esfuerzo lo suficiente (aumenta 4 puntos).

- Ítem 3. Me es fácil persistir en lo que me he propuesto hasta llegar a alcanzar mis metas. (aumenta 3 puntos). - Ítem 4. Tengo confianza en que podría manejar eficazmente acontecimientos inesperados (aumenta 4 puntos). 


\section{Discusión}

A raíz de las puntuaciones obtenidas, se puede confirmar que se cumple la hipótesis planteada, ya que la intervención realizada con la aplicación del modelo de coaching "La Jirafa de Cantón", ha sido eficaz, dado que los resultados han declarado un efecto positivo en las variables psicológicas medidas, concretamente en la Autoestima, algunas dimensiones de Bienestar Psicológico (Relaciones Positivas, Dominio del Entorno y Propósito en la Vida) y la percepción de Autoeficacia General.

Además, al preguntar a la jugadora por la utilidad de las sesiones de coaching, la valoración fue "positiva y útil, me ayudó a darme cuenta de la posibilidad de optimizar mi rendimiento". Con el uso de los propios recursos que tiene, puede desarrollar ciertas pautas en las sesiones de entrenamiento o en la competición, que faciliten la consecución de los objetivos personales a la vez que "me he dado cuenta del alcance de mis deficiencias, y que puedo hacer para controlarlo. Así poder conseguir mi objetivo".

Tras finalizar la intervención, sigue teniendo ficha federativa en la misma categoría y en el mismo club pero, según afirma, ha finalizado la temporada aumentando la cantidad de minutos jugados en la competición como en el torneo post-temporada, según su propia percepción y los registros llevados a cabo por el entrenador del equipo. Este hecho le permite percibir un mayor número de oportunidades para mostrar sus recursos y así, optimizar su rendimiento en las situaciones críticas.

Estos resultados están en la línea de otras intervenciones de coaching (García-Naveira, 2013, 2016; García-Naveira et al., 2017; Peris-Delcampo, 2015; Peris-Delcampo et al., 2016). Estos autores llegan a conclusiones similares, por tanto, cuando una intervención en coaching aporta mejoras en la vida de una persona, es posible que se favorezca a la mejora psicológica (en este caso, bienestar psicológico, autoestima y autoeficacia). Así, se desprende de los resultados de este estudio que se debe considerar el ámbito del coaching como una estrategia de intervención psicológica en modificación de la conducta que puede tener una cierta eficacia en la mejora percibida de los deportistas, claramente establecida para las personas licenciadas o graduadas en Psicología.

A modo de conclusión, dentro del perfil profesional del psicólogo del deporte (Cantón, 2016), intervenciones y estudios de este tipo son importantes, a nivel aplicado, por dos importantes razones: por un lado porque ofrece herramientas de trabajo a los especialistas en Psicología del Deporte que, de manera fundamentada desde la Psicología, y con relativamente pocas sesiones, puede hacer una intervención eficaz; y por otro, la sistematización y evaluación de este tipo de intervenciones ofrece elementos de análisis respecto al protocolo de actuación y al modelo de la "Jirafa de Cantón" para ir potenciándolo y mejorándolo si procede.

En la línea planteada por García-Naveira et al. (2017), dentro de las limitaciones en el estudio destacar que para garantizar la no influencia de variables contaminadoras como el género, la edad, el número de sesiones y horas de aplicación del programa, o las que pueden generar el propio psicólogo que ha intervenido, hubiese sido necesario demostrar que estas variables no influyen sobre el resultado. Tampoco se ha realizado un seguimiento posterior a la última sesión del proceso de coaching, por lo que no se tienen datos de que si estos cambios se mantuvieron a lo largo del tiempo. Por último, mencionar la necesidad de seguir estudiando las diferentes teorías y modelos psicológicos que pueden estar implicados en el coaching con el objetivo de profundizar sobre esta temática.

\section{Referencias}

Atkinson, J. W. (1957). Motivational determinants of risk-taking behavior. Psychological Review, 64, 359-372. https://doi. org/10.1037/h0043445

Atkinson, J. W. (1983). Personality, motivation and action. Nueva York, Estados Unidos: Praeger.

Bandura, A. (1977). Social learning theory. Englewoods Cliffs, NJ, Estados Unidos: Prentice-Hall.

Bandura, A. (1982). Self-efficacy mechanism in human agency. American Psychologist, 37, 122-147. https://doi.org/10.1037/0003-066X.37.2.122

Bandura, A. (1997). Self-efficacy: The exercise of control. Nueva York, NJ, Estados Unidos: Freeman and Co. Publishers.

Buceta, J. M. (2016). Habilidades del coach. Madrid, España: Universidad Nacional de Educación a Distancia.

Cantón, E. (2013). La "Jirafa de Cantón": un modelo de estrategia motivacional aplicado desde la perspectiva del coaching. Granada, España: Editorial CSV.

Cantón, E. (2014a). 'Cantón's Giraffe': A motivational strategy model applied from the perspective of coaching. The Coaching Psychologist, 10 (1), 26-34

Cantón, E. (2014b). ¿El coaching es psicología? ¿Quién puede aplicar el Coaching y en qué entornos? Informació Psicològica, 107, 2-10. http://dx. medra.org/10.14635/IPSIC.2014.107.1

Cantón, E. (2016). La especialidad profesional en Psicología del Deporte. Revista de Psicología Aplicada al Deporte y al Ejercicio Físico, 1(e2), 1-7. https://doi.org/10.5093/rpadef2016a2

Cantón, E. y Peris-Delcampo, D. (2017, julio). Psychological intervention in sport from the motivational coaching perspective. Comunicación presentada en el 14th World ISSP Congress of Sport Psychology, Sevilla, España.

Caperán Vega, J. A. y Peris-Delcampo, D. (2014). Del coaching ejecutivo a otros campos de aplicación: una opción para optimi- 
zar el trabajo del psicólogo. Informació Psicològica, 107, 22-33. http://dx.medra.org/10.14635/IPSIC.2014.107.3

De la Vega, R., Ruiz-Barquín, R., Batista, F., Ortín, F. J. y Giesenow, C. (2012). Effects of feedback on self-efficacy expectations based on the athlete's optimistic profile. Psychology, 23(12A), 1208-1214.

Deci, E.L. y Ryan, R.M. (1985). The general causality orientations scale: Self-determination in personality. Journal of Research in Personality, 19, 109-134.

Díaz, D., Rodríguez-Carvajal, R., Blanco, A., Moreno-Jiménez, B., Gallardo, I., Valle, C. y Van-Dierendonck, D. (2006). Adaptación española de las escalas de bienestar psicológico de Ryff. Psicothema, 18, 572-577.

Dweck, C. S. (1991). Self-theories and goals: Their role in motivation, personality and developement. En R. A. Deinstbier (Ed.): Nebraska Symposium on Motivation 1990 (pp. 199-235). Lincoln, Estados Unidos: University of Nebraska Press.

Ford, M. E. (1992). Motivation humans: Goal, emotions and personal agency beliefs. Newbury Park, CA, Estados Unidos: Sage.

Frías, D., Pascual, J. y García, F. (1999). Principios del diseño de investigación. Valencia, España: Editorial CSV.

Gallwey, T. (2006). El juego interior del tenis. Málaga, España: Editorial Sirio, S.A.

García-Naveira, A. (2011). Aproximación al empleo profesional del coaching en el deporte. Informació Psicològica, 101, 26-39.

García-Naveira, A. (2013). Aplicación profesional del coaching en el deporte: un estudio de caso único. Cuadernos de Psicología del Deporte, 13(2), 101-112.

García-Naveira, A. (2016). Percepción del bienestar y de la salud psicológica, y la eficacia de un programa de intervención en coaching en deportistas de rendimiento. Revista Iberoamericana de Psicología del Ejercicio y el Deporte, 17(2), 211-219.

García-Naveira, A., García-Mas, A., Ruiz-Barquín, R. y Cantón, E. (2017). Programa de intervención basada en el coaching en jóvenes deportistas de alto rendimiento, y su relación con la percepción de bienestar y salud psicológica. Revista de Psicología del Deporte, 26(2), 37-44.

Keyes, C. L. (2002). The mental health continuum: From languishing to flourishing in life. Journal of Health and Social Behavior, 43, 207-222. https://doi.org/10.2307/3090197

León, E., Boix, S., Serrano, M. A. y Paredes, N. (2017). Motivación y toma de decisiones en Voleibol: Influencia de los años de experiencia. Revista de Psicología Aplicada al Deporte y al Ejercicio Físico, 1(e4), 1-10. https://doi.org/10.5093/rpadef2017a4

Locke, E. A. (1968). Toward a theory of task motivation and incentives. Organizational Behavior and Human Performance, 3, 157189.

Locke, E. A., y Latham, G. P. (1990). A theory of goal setting and task performance. Englewoods Cliffs, NJ, Estados Unidos: Prentice-Hall.

McClelland, D. C. (1989). Estudio de la Motivación Humana. Madrid, España: Narcea.

Ortín, F. J. (2017). El coaching desde el enfoque positivo de la Psicología. Valencia, España: Certificado Universitario en Psicología Positiva y Coaching de ADEIT -Universidad de Valencia.

Palmero, F., Fernández-Abascal, E., Martínez, F. y Chóliz, M. (2001). Psicología de la Motivación y Emoción. México, México: McGrawHill.
Peris-Delcampo, D. (2011a). ¿Qué es el coaching? Valencia: Máster en Coaching y Liderazgo de ADEIT-Universidad de Valencia.

Peris-Delcampo, D. (2011b). Aspectos que favorecen el proceso del coaching. Valencia: Máster en Coaching y Liderazgo de ADEIT-Universidad de Valencia.

Peris-Delcampo, D. (2014). El coaching: cómo podemos utilizarlo con éxito. Informació Psicològica, 107, 11-22. https://doi. org/10.14635/IPSIC.2014.107.2

Peris-Delcampo, D. (2015, junio). Trabajo psicológico a distancia con entrenadores y jugadores profesionales de fútbol. Comunicación presentada en el I Congreso Internacional sobre Psicología y Fútbol, Santiago de Compostela, España.

Peris-Delcampo, D. (2016). Nociones básicas de psicología del deporte y coaching para gestores de empresas deportivas. Almería, España: Círculo Rojo.

Peris-Delcampo, D. (2017, julio). Key points for the efficient coaching application as a tool for the psychological intervention in sport. Comunicación presentada en el 14th World ISSP Congress of Sport Psychology, Sevilla, España.

Peris-Delcampo, D., Expósito, V. E. y Cantón, E. (2016). Intervención psicológica en natación adaptada desde la perspectiva del coaching motivacional. Informació Psicològica, 112, 59-73. https://doi.org/10.14635/IPSIC.2016.172.5.

Reeve, J. (2010). Motivación y emoción (5ª ed.). México, México: McGraw-Hill.

Rosenberg, M. (1965). La autoimagen del adolescente y la sociedad (traducción de 1973). Buenos Aires, Argentina: Paidós

Rosenberg, M. (1989). Society and the adolescent self-image. Middletown, CT, España: Wesleyan University Press.

Ryan, R. M. y Deci, E. L. (2001). To be happy or to be self-fulfilled: A review of research on hedonic and eudaimonic well-being. En S. Fiske (Ed.), Annual Reiew of Psychology (Vol. 52; pp. 141-166). Palo Alto, CA, Estados Unidos: Annual Reviews, Inc.

Ryan, R.M., Hunta, V. y Deci, E.L. (2008). Living well: a self-determination theory perspective on eudaimonia. Journal of Hapiness Studies, 9, 139-170.

Ryff, C. (1989a). Beyond Ponce de Leon and Life satisfaction: New directions in quest of successful aging. International Journal of Behavioral Development, 12, 35-55. https://doi. org/10.1177/016502548901200102

Ryff, C. (1989b). Happiness is everything, or is it? Explorations on the meaning of psychological well-being. Journal of Personality and Social Psychology, 57, 1069-1081. https://doi. org/10.1037/0022-3514.57.6.1069

Ryff, C., y Singer, B. (2002). From social structure to biology. En C. Snyder y A. López (Eds.), Handbook of positive psychology (pp. 63-73). Londres, Reino Unido: Oxford University Press.

Sanjuán, P., Pérez-García, A. M. y Bermúdez, J. (2000). Escala de autoeficacia general: datos psicométricos de la adaptación para la población española. Psicothema, 12, 509-513.

Seligman, M. E. P. (1999). The President's Adress. APA 1998 Annual Repport. American Psychologist, 54, 559-562.

Seligman, M. E. P. (2002). Authentic Happiness: Using the New Positive Psychology to Realize Your Potential for Lasting Fulfillment. New York, Estados Unidos: Free Press/Simon \& Schuster.

Seligman, M.E.P. y Csikszentmihalyi, M. (2000). Positive psychology: An introduction. American Psychologist, 55, 5-14. 
Schwarzer, R. y Baessler, J. (1996). Evaluación de la autoeficacia: adaptación española de la escala de Autoeficacia General. Ansiedad y Estrés, 2, 1-8.

Schwarzer, R. y Jerusalem, M. (1995). General self-efficacy scale. En J. Weinman, S. Wright y M. Johnston (Eds.). Measures in health psychology: A user's portfolio. Causal and control belief (pp. 35-37). Windsor, Reino Unido: NFER-NELSON.

Tolman, E.C. (1955). Principles of performance. Psychological Review, 62, 315-326.

Vázquez-Morejón, A.J., Jiménez, R. y Vázquez-Morejón, R. (2004). Escala de Autoestima de Rosenberg: fiabilidad y validez en población clínica española. Apuntes de Psicología, 22, 247-255.

Vázquez Morejón, A. J., Vázquez-Morejón Jiménez, R. y Bellindo Zanin, G. (2013). Fiabilidad y validez de la Escala de Autoesti- ma de Rosenberg (EAR) en pacientes con diagnóstico de psicosis. Apuntes de Psicología, 37, 37-43.

Vera, B. (2008). Psicología positiva. Una nueva forma de entender la psicología. Madrid, España: Calamar Ediciones.

Villa, J. P. y Caperán, J. A. (2010). Manual de Coaching. Barcelona, España: Profit Editorial.

Vroom, V. H. (1964), Work and Motivation. New York, NY, Estados Unidos: John Wiley and Sons.

White, R. W. (1959). Motivation Reconsidered: The concept of competence. Psychological Review, 66, 297-333.

Whitmore, J. (2003). Coaching el método para mejorar el rendimiento de las personas. Barcelona, España: Editorial Paidós.

\section{Anexo 1}

\section{Preguntas utilizadas para la identificación del modelo de "La Jirafa de Cantón"}

\section{META}

\section{Preguntas generales sobre la meta}

¿Cuál es tu objetivo ahora mismo?

¿Qué quieres conseguir exactamente?

\section{Específicas y operacionalizadas}

¿Crees que es específica? ¿La consideras concreta, es decir, un aspecto concreto de tu vida referido al futbol sala o algo más general válido para otras cosas que quieras alcanzar?

¿Crees que es específica? ¿La consideras concreta, es decir, un aspecto concreto de tu vida referido al futbol sala o algo más general válido para otras cosas que quieras alcanzar?

\section{Difícil, posible pero improbable}

¿Consideras que este objetivo es realista?

\section{Orientadas a la tarea}

¿Qué estás dispuesto a hacer para lograr tu objetivo?

\section{Establecerlas a corto/largo plazo}

¿Crees que es mejor dividir esta meta en varias partes?

¿Crees que hacer una especie de escalera e irle añadiendo escalones es la mejor forma de alcanzar tu meta?

\section{Favorecer metas de rendimiento frente a metas de resultado}

¿Crees que sería bueno aumentar tu nivel de competencias?, ¿Podrías plantarte metas relacionadas con tu nivel de futbol sala?

\section{Elaborarlas en positivo}

¿Qué sería para ti aprovechar el tiempo? 


\section{Fijar Fechas}

¿Cómo vas a organizarte de cara al futuro?

¿Hace cuánto te planteaste esta meta?

¿Qué te propones conseguir en tres jornadas?

¿Te planteas un plazo mínimo para conseguirlo o es una meta a largo plazo?

¿Cuándo quieres alcanzar esta meta? ¿Para cuándo te planteas el cambio?

\section{Anotarlas y registrarlas}

¿Has pensado en utilizar algún sistema de autoresgistro para ir viendo cómo va tu meta y en qué medida se está o no cumpliendo?

¿Sabrías hacerte alguna hoja de registro o anotar estos datos? ¿Conoces a alguien que pudiera echarte una mano con esto?

\section{Estrategias de logro}

¿Qué recursos crees que tienes para conseguir esta meta?

¿Dónde puedes obtener estos recursos?

\section{Flexible y ajustable intrapersonalmente}

¿Crees que puede obtener esta meta en el tiempo que te has dado?

¿Crees que podrías necesitar algún escalón alternativo en tu escalera?

\section{Favorecer el compromiso desarrollando estrategias motivacionales}

¿Qué necesitas para obtener esta meta?

¿Te sientes igual de motivado desde que te planteaste la meta hasta ahora?

¿Estarías dispuesto a trabajar y mejorar tu rendimiento para obtener esta meta?

\section{Promover el apoyo social}

¿Te sientes apoyarlo con este reto o meta?

¿Qué apoyos tienes, quien te ayuda para lograrla?

\section{Evaluarlas en los momentos fijados}

¿Tienes alguna manera de obtener información sobre el cumplimiento de tu meta?

¿Qué datos puedes utilizar y anotar para ver si tu meta se está logrando?

¿Podrías anotar y registrar tu nivel de progreso? ¿Cómo? 